\title{
Equation of State Data for Iron at Pressures beyond 10 Mbar
}

\author{
D. Batani, ${ }^{1}$ A. Morelli, ${ }^{1}$ M. Tomasini,${ }^{1}$ A. Benuzzi-Mounaix,${ }^{2}$ F. Philippe, ${ }^{2}$ M. Koenig, ${ }^{2}$ B. Marchet,${ }^{3}$ I. Masclet, ${ }^{3}$ \\ M. Rabec, ${ }^{3}$ Ch. Reverdin, ${ }^{3}$ R. Cauble ${ }^{4}$ P. Celliers,${ }^{4}$ G. Collins,${ }^{4}$ L. Da Silva,${ }^{4}$ T. Hall,${ }^{5}$ M. Moret, ${ }^{6}$ B. Sacchi, ${ }^{7}$ \\ P. Baclet, ${ }^{8}$ and B. Cathala ${ }^{9}$ \\ ${ }^{1}$ Dipartimento di Fisica “G. Occhialini,” Università di Milano-Bicocca and INFM, Piazza della Scienza 3, 20126 Milano, Italy \\ ${ }^{2}$ Laboratoire pour l'Utilisation des Lasers Intenses (LULI), UMR n. 7605 CNRS-CEA-X-Paris VI, Ecole Polytechnique, \\ 91128 Palaiseau, France \\ ${ }^{3}$ Commissariat à l'Energie Atomique, CEA/DIF, B.P. 12, 91680 Bruyères-le-Châtel, France \\ ${ }^{4}$ Lawrence Livermore National Laboratory (LLNL), Livermore, California \\ ${ }^{5}$ University of Essex, Wivenhoe Park, Colchester, Essex CO4 3SQ, United Kingdom \\ ${ }^{6}$ Dipartimento di Scienza dei Materiali, Università di Milano-Bicocca, Via Cozzi, 20126 Milano, Italy \\ ${ }^{7}$ Dipartimento di Chimica Fisica ed Elettrochimica, Università di Milano, Via Golgi 19, 20133 Milano, Italy \\ ${ }^{8}$ Commissariat à l'Energie Atomique, CEA/CVa, Valduc, 21120 Is-sur-Tisle, France \\ ${ }^{9}$ Commissariat à l'Energie Atomique, CEA/CESTA (Bordeaux), B.P. 2, 33114 Le Barp, France
}

(Received 9 November 2001; published 22 May 2002)

\begin{abstract}
We present equation of state points for iron, in the pressure range 10-45 Mbar, the first obtained with laser-driven shock waves. The experiment has been performed with the high energy laser Phebus, optically smoothed with Kinoform phase plates. Our results double the set of existing experimental data at very high pressures showing good agreement with the predictions of the quotidian equation of state model and with previous results.
\end{abstract}

DOI: 10.1103/PhysRevLett.88.235502

PACS numbers: $62.50 .+\mathrm{p}, 52.38 .-\mathrm{r}$

Introduction.- The knowledge of the equation of state (EOS) of iron at extreme pressures is of interest for several fields of science. First, iron is the main component of the Earth's core, where pressures between 1.3 and 3.7 Mbar are reached. The knowledge of iron EOS is then essential to the construction of a realistic model of Earth and in particular the description of the transition between the liquid outer core and the solid inner core. Many recent works have been devoted to the structure of Earth's core and the iron EOS in geophysical context [1-9]. Iron EOS at pressures higher than reached inside the Earth is also interesting for many reasons. First, EOS data at pressures higher than 3.7 Mbar put constraints in the EOS at lower pressures and are hence useful to elaborate a model of iron at "geophysical" pressures. Second, the current idea on the formation of the Earth-moon system is based on the collision between two celestial bodies [10] during which very high pressures (up to several tents Mbar) would have been produced. Third, during the accretion process, which originated the Earth core, collisions among several planetesimals may have also implied shock waves with very high pressures [11]. Iron is also found, possibly differentiated, in the rocky cores of intermediate planets such as Uranus and Neptune [12]. Finally, telluric planets with mass bigger than the Earth, and internal pressure exceeding 3.7 Mbar, might be found among the many recently discovered extrasolar planetary systems.

Despite such large interest, not many experimental data are available above 10 Mbar where a total of 9 iron EOS points is reported in scientific literature, for iron at normal initial density [13-21]. Possibly also as a result of this lack of data, various models predict rather different behaviors at very high pressures. For instance, according to the wellknown quotidian equation of state (QEOS) model [22], the compressibility of iron at $40 \mathrm{Mbar}$ is $\approx 2.7$, substantially smaller than predicted by the SESAME tables [23]. Available data seem in closer agreement with QEOS, however, no definite conclusions can be drawn due to the few experimental points.

Such lack depends on the difficulty of creating high compressions in the laboratory, while measuring the relevant parameters. Pressures above a few Mbar are obtained only by dynamical methods (shock waves), which in the past were produced, in the case of iron, with two-stage light-gas guns, and with chemical and nuclear explosions [1,8,13-21].

Recently, laser-driven shocks have become a reliable tool in high pressure physics thanks to developments in control of uniformity, steadiness, and preheating [24-27], and have been used for EOS measurements of deuterium [28], copper [29], plastic [30], and gold [31].

In this paper, we present the first experimental data on iron obtained with laser-driven shocks in the pressure range 10-45 Mbar. The experiment was performed at the CEA Laboratory in Limeil-Valenton, with the high-energy laser Phébus, and 9 new iron EOS points were obtained, doubling the existing data set.

The experiment.-The experiment uses the impedance mismatch method $[24,32]$ based on the simultaneous measurement of the shock velocity $D$ in two different materials. The first one is the material with unknown EOS (iron in our case), while the second one is a reference material; we have chosen aluminum, because its EOS is well known at high pressures [23]. 
The method requires "two steps-two materials" targets made of a common base of the reference material ( $\mathrm{Al})$ and two steps: the first of Al, the second of iron (Fig. 2, top). The laser irradiates the base where a polypropylene $(\mathrm{CH})$ layer is deposited to reduce preheating, i.e., the fact that $\mathrm{x}$ rays, produced during laser interaction, penetrate into the material ahead of the shock. The use of $\mathrm{CH}$ reduces the amount of $\mathrm{x}$ rays and produces softer, hence less penetrating $\mathrm{x}$ rays, strongly reducing preheating as demonstrated in previous experiments [27].

Targets were fabricated at the CEA Micro-Targets Laboratory. $\mathrm{Al}$ was machined to directly obtain the base and the step, while iron was glued on the base with a thin layer of PVA (polyvinyl alcohol at 10\%) glue after laser cutting of a Fe foil. Typical dimensions were $30 \mu \mathrm{m}(\mathrm{CH}), 80 \mu \mathrm{m}$ (Al base), $20.5 \mu \mathrm{m}$ (Al step), and $19 \mu \mathrm{m}$ (Fe step). The base and step were measured with a profilometer (Dektak) and a SEM microscope, before and after gluing to assure that the glue thickness was negligible $(\leq 1 \mu \mathrm{m})$.

Using this target design we could measure the shock velocities $D_{\mathrm{Al}}$ and $D_{\mathrm{Fe}}$ on the same laser shot. The knowledge of $D_{\mathrm{Al}}$ and of the Al EOS provides the shock pressure $P$ and, through Hugoniot-Rankine relations, all the other physical quantities in aluminum. Hence, by considering that the Al-Fe interface is in dynamic equilibrium, and by applying the conditions for shock transmission into a denser material (impedance mismatch), it is possible to obtain an EOS point for iron, once the shock velocity $D_{\mathrm{Fe}}$ is measured. This is a classical method in EOS experiments, described in textbooks [32] and used with conventional methods of dynamic compression and more recently with laser-driven shock waves [24]. The scheme of the experimental setup is shown in Fig. 1.

In our experiment the high-energy Phébus $\mathrm{Nd}$ laser was used, converted to the second harmonic $(\lambda=0.526 \mu \mathrm{m})$, giving shots with energy between 0.6 and $2.5 \mathrm{~kJ}$ in a square 4 ns pulse. The focal spot was $700 \times 600 \mu \mathrm{m}^{2}$, giving intensities in the range $10^{13}-10^{14} \mathrm{~W} / \mathrm{cm}^{2}$. Kinoform phase plates were used to produce a flat irradiation profile and eliminate large-scale laser hot spots [33]. This assures the

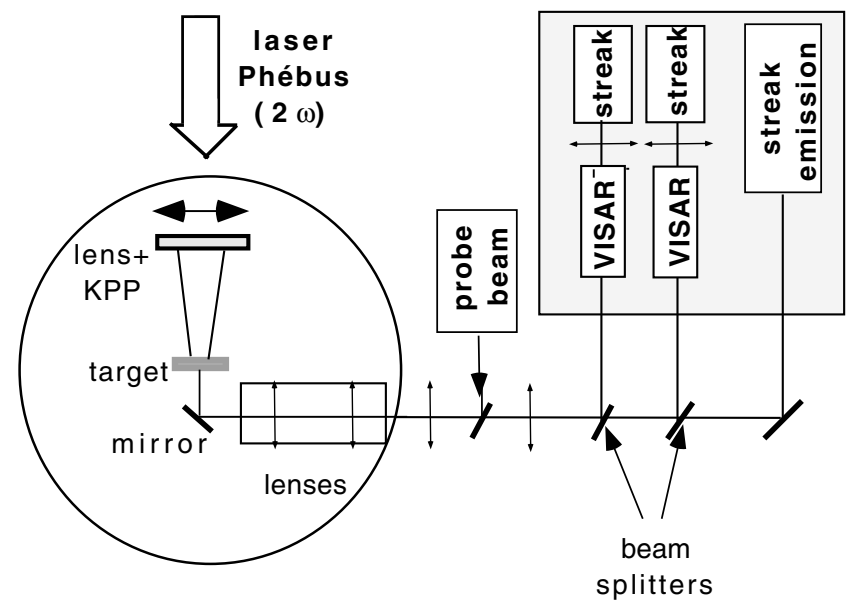

FIG. 1. Experimental setup. uniformity of laser energy deposition on target, a crucial point in allowing the generation of planar shocks which can be used as a reliable tool in EOS experiments [24-26].

Two independent diagnostics were used to measure the shock breakout time from the base and from the two steps of the target rear side, and find the shock travel time in steps and the shock velocity (step thickness being measured before the shot).

The first is based on target self-emission: the arrival of the shock at the target rear face causes a strong increase in temperature and emission, which is recorded by a visible streak-camera resolving the image in space and time (Fig. 2). The magnification of the optical system was $\times 13$, the spatial resolution of the diagnostic $8 \mu \mathrm{m}$ and the temporal resolution $20 \mathrm{ps}$.

The second diagnostics is a VISAR interferometer (velocity interferometer system for any reflector) [34]: a 10 ns probe beam $(\lambda=1.064 \mu \mathrm{m})$ of energy $\approx 1 \mathrm{~mJ}$ is reflected from the rear side of the target; the reflected beam is sent to the VISAR and then to a streak camera which produces spatially and temporarily resolved reflection images (Fig. 3). The reflectivity drop corresponds to the shock breakout. Again this allows $D_{\mathrm{Al}}$ and $D_{\mathrm{Fe}}$ to be measured. In this case the magnification of the optical system was $\times 26$, giving a spatial resolution of $4 \mu \mathrm{m}$ while the temporal resolution was $40 \mathrm{ps}$.

The purpose of having a VISAR instead of a simpler time-resolved reflectometry diagnostics was that it allowed

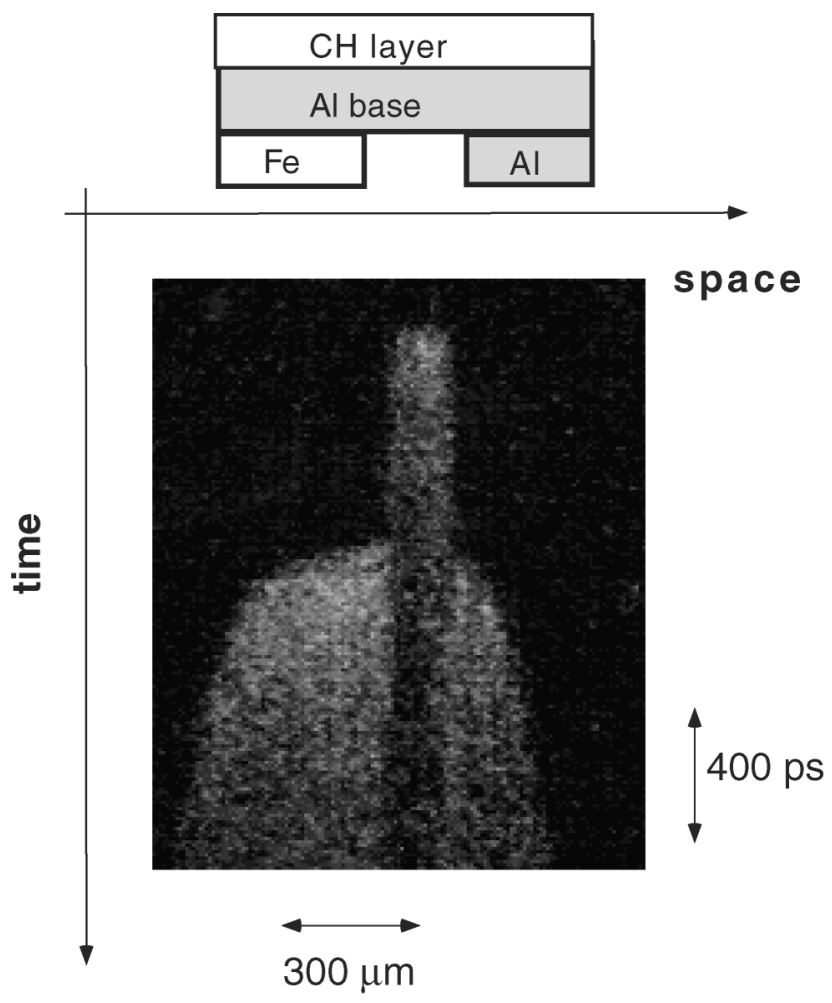

FIG. 2. Streak image of the target rear side in emission. Shot energy was $0.6 \mathrm{~kJ}$. Shock break-out from the Al step on the left and from the Fe step on the right. The schematic of two steps-two materials target is shown on the top. 


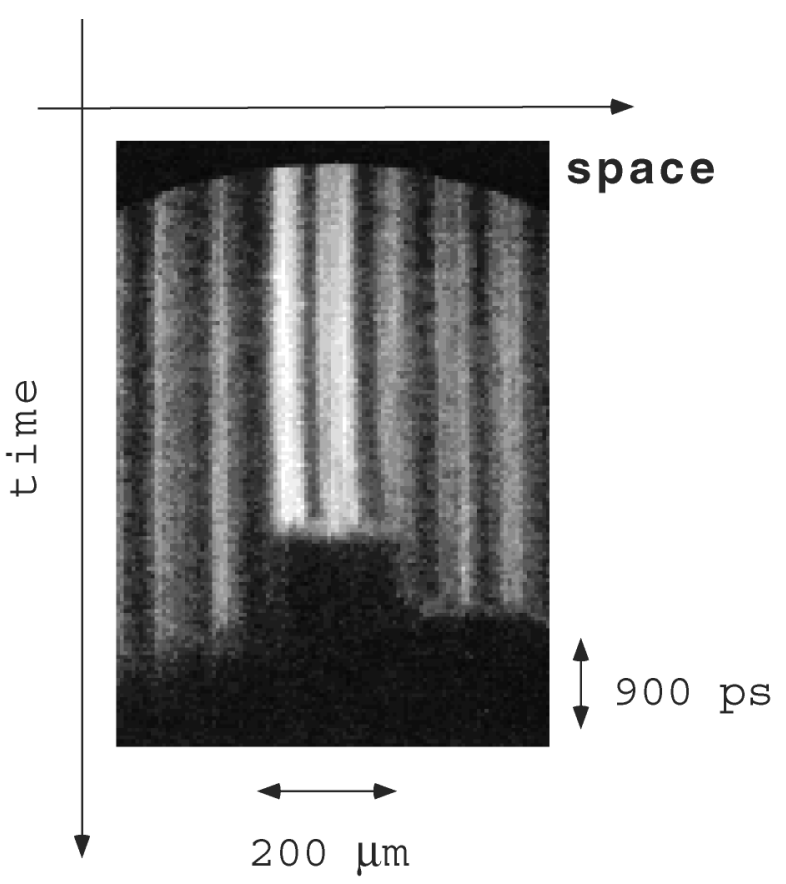

FIG. 3. VISAR image of the target rear surface in reflection. Energy of the main beam $1 \mathrm{~kJ}$.

us to detect any motion of the rear target surface before shock breakout. This motion results in a Doppler shift of the reflected beam and hence in a fringe shift in the experimentally recorded images. Since such motion is related to target preheating [27,34], we verified that the rear side preheating was small on most shots, as discussed later. In our case the interferometer employs the Mach-Zender configuration and we used two VISARs with different sensitivities: 7 and $21 \mathrm{~km} \mathrm{~s}^{-1}$ fringes ${ }^{-1}$.

Another requirement for applying the impedance matching method is a steady shock. Computer simulations, corresponding to our experimental parameters, were performed with the hydrocode MULTI [35] and showed that the shock speed is constant within $\pm 1.1 \%$, a small value when compared to our error bars (see over). Experimentally, the streak image in Fig. 2 gives some evidence of steadiness since luminosity at shock breakout is related to temperature (i.e., shock pressure and velocity) and is about the same on the Al base and the Al step (of course, after breakout, luminosity rapidly drops down due to material expansion and cooling). A more direct experimental evidence of steadiness can be obtained with the VISAR diagnostics using transparent targets. A preliminary analysis of data obtained in the same experiment on water targets [36] is in agreement with results of computer simulations.

Finally, the impedance matching method requires shock planarity. Figures 2 and 3 show some curvature of the shock front at the edges, as caused by the finite spot size of the laser. Measurements were performed only on the central part of the image where, thanks to the use of KPPs, the shock front is planar.

Results and discussion.-Figure 4 shows our nine experimental points for iron EOS, in a $\left(\rho / \rho_{0}, P\right)$ diagram,

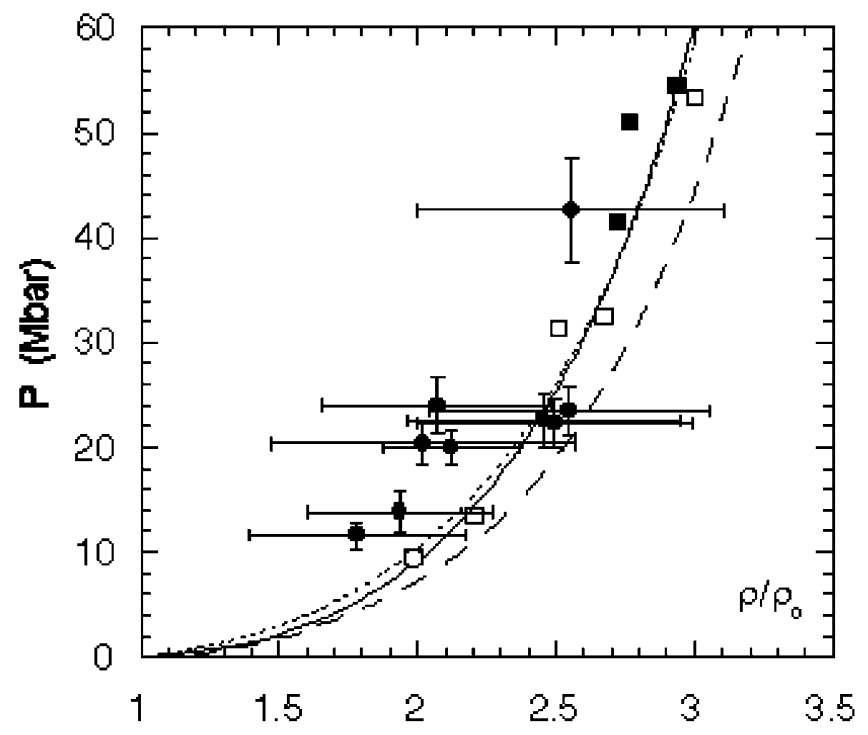

FIG. 4. Experimental results for iron EOS. Curves correspond to the QEOS model, continuous line [22]; the SESAME tables, dashed line [23] and the empirical fit given by Trunin, dotted line [37]. Experimental points correspond to the present work, black circles, Al'tshuler, white squares [13-15], Krupnikov, white circles [16], and Trunin, black squares $[17,18,19]$. Not shown a point by Trunin at 104.8 Mbar.

where $\rho_{0}=7.85 \mathrm{~g} / \mathrm{cm}^{3}$ is the density of cold iron. The experimentally achieved pressure range is 10-45 Mbar. Also shown in Fig. 4, the Hugoniot curves derived from the SESAME tables and the QEOS model, and the empirical fit by Trunin [37]. We have considered this, among the various fits present in literature (see Refs. [13,20,21], for instance) because it gives a better comparison with existing Fe EOS points in the Megabar pressure range. It gives a linear relation between $D$, the shock velocity, and $U$, the fluic velocity: $D=(6.41+1.213 U)$, where both $D$ and $U$ are in $\mathrm{km} / \mathrm{s}$, and it is very close to the QEOS model.

Errors are about $10 \%$ on pressure, and about $20 \%$ on compression. Error bars have been estimated by calculating the propagation of the experimental error on the shock velocity $(\approx 5 \%)$ on the quantities determined by the mismatch method. The error on shock velocity is determined from the uncertainties on step thickness and from the streak-camera temporal resolution (determined by the streak camera sweep speed and slit size), and it also includes data reading errors. [It is worth noting that density is the quantity which is determined with the largest error from Hugoniot-Rankine relations.]

As can be seen from Fig. 4, all our points are above the SESAME curves, while the agreement is much better with the QEOS model and with the semiempirical fit by Trunin (given our error bars these are compatible with all our experimental points); in fact, by calculating the $\chi^{2}$ we obtain a $\chi^{2} \approx 3.2$, or $a \geq 95 \%$ compatibility with these curves. The compatibility with SESAME is definitely smaller. As already stated, this implies a compressibility of iron $\approx 2.7$ at 40 Mbar, substantially smaller than predicted by SESAME [23], i.e., $\approx 3$. 
Finally, it is necessary to discuss the problem of preheating. In laser-shock experiments this may lead to inaccurate measurements, because it modifies the material properties before shock arrival, making it impossible to properly apply the mismatch method. With the VISAR, the shift of interference fringes allows us to evaluate the expansion velocity due to the preheating of the target before the shock arrival. As previously said, this is absent on most of the shots. Since the sensitivity of the system is $7 \mathrm{~km} \mathrm{~s}^{-1}$ fringes ${ }^{-1}$, and since we are able to detect a shift corresponding to a $1 / 15$ th of fringe, velocities as small as $0.4 \mathrm{~km} / \mathrm{s}$ can be detected.

On some shots (corresponding, as expected, to higher laser energies) there was some preheating, especially on the target base, as seen in Fig. 3 (x rays which cause preheating are absorbed in the thicker steps before reaching the target rear side). In those, expansion velocities up to $3.7 \mathrm{~km} / \mathrm{s}$ were measured on the Al base.

In order to establish a relation between preheating and expansion velocity, we used MULTI simulations. These showed how a velocity of $3.7 \mathrm{~km} / \mathrm{s}$ corresponds to a preheating temperature of $\approx 1.2 \mathrm{eV}$ which largely exceeds both the melting and the boiling temperatures for $\mathrm{Al}$ (660 and $2467{ }^{\circ} \mathrm{C}$, respectively). Hence the material on rear side is vaporized and expands with a velocity of the order to the sound velocity, close indeed to the expansion velocity determined with MULTI simulations. The material expansion is less than a couple of microns during the typical observation time, which explains why a high reflectivity is maintained on the rear side even if the material is vaporized and characterized by a density profile.

Such preheating did not substantially affect our experimental results, as we verified with a simple model [38], since the expected variations of $D, P$, and $\rho / \rho_{0}$ induced by preheating are within our (quite large) measurement error bars. Also, the fact that experimental points obtained with completely different methods (lasers, gas guns, chemical and nuclear explosions) converge around a unique curve, strongly suggests that indeed preheating does not have a large effect, as its level is a priori expected to be completely different with different experimental setups.

Conclusions.-In conclusion, in this Letter we have reported new EOS data for iron at pressures higher than 10 Mbar. These are the first results obtained with laserdriven shocks and double the set of existing data in this pressure range. Our points are in fair agreement with previously available results obtained with conventional methods and nuclear explosions and with the analytical fit proposed by Trunin [37]. Also, the agreement with the QEOS theoretical model [22] is quite good and better than with the well-known SESAME tables [23], implying a reduced compressibility of iron at high pressures.

The work was supported by the European Union TMR program, Contract No. ERBFMGECT 950016. A. M. took part in the experiment with a Socrates grant. We also ac- knowledge useful discussions with F. Guyot, IPG, France. Finally, we acknowledge the support of the CNR/CNRS programme on "Laboratory astrophysics with high power lasers."

[1] O. L. Anderson and Al Duba, J. Geophys. Res. 102, 659 (1997).

[2] A. Laio et al., Science 287, 1027 (2000).

[3] R. Lubbers et al., Science 287, 1250 (2000).

[4] G. Fiquet et al., Science 291, 468 (2001).

[5] S. Merkel et al., Science 288, 1626 (2000).

[6] S. C. Singh et al., Science 287, 2471 (2000).

[7] K. Mao et al., Science 292, 927 (2001).

[8] R. Boehler, Rev. Geophys. 38, 221 (2000).

[9] C. S. Yoo et al., Science 275, 94 (1997).

[10] A. G. W. Cameron, Icarus 126, 126 (1997).

[11] D. J. Stevenson, Science 214, 611 (1981).

[12] W. B. Hubbard et al., Science 253, 648 (1991).

[13] L. V. Al'tshuler et al., Zh. Prikl. Mekh. Tekh. Fiz. 2, 3 (1981) [J. Appl. Mech. Techn. Phys. 22, 145 (1981)].

[14] L. V. Al'tshuler et al., Zh. Eksp. Teor. Fiz. 54, 785 (1968) [Sov. Phys. JETP 27, 420 (1968)].

[15] L. V. Al'tshuler et al., Zh. Eksp. Teor. Fiz. 72, 317 (1977) [Sov. Phys. JETP 45, 167 (1977)].

[16] K. K. Krupnikov et al., Dokl. Akad. Nauk SSSR 148, 1302 (1963) [Sov. Phys. Dokl. 8, 205 (1963)].

[17] R.F. Trunin et al., Zh. Eksp. Teor. Fiz. 62, 1043 (1972) [Sov. Phys. JETP 35, 550 (1972)].

[18] R. F. Trunin et al., Zh. Eksp. Teor. Fiz. 102, 1433-1438 (1992) [Sov. Phys. JETP 75, 777 (1992)].

[19] R. F. Trunin et al., Zh. Eksp. Teor. Fiz. 103, 2189 (1993) [Sov. Phys. JETP 76, 1095 (1993)].

[20] J. M. Brown and R. G. McQueen, J. Geophys. Res. 91, 7485-7494 (1986).

[21] LASL Shock Hugoniot Data, edited by S. P. Marsh (University of California Press, Berkeley, 1980).

[22] R. M. More et al., Phys. Fluids 31, 3059 (1988).

[23] K. S. Holian, LANL Report No. LA-10160-MS UC-34, 1984.

[24] M. Koenig et al., Phys. Rev. Lett. 74, 2260 (1995); Th. Lower et al., Phys. Rev. Lett. 72, 3186 (1994).

[25] D. Batani et al., Europhys. News 27, 210 (1996).

[26] Y. M. Gupta and S. M. Sharma, Science 277, 909 (1997).

[27] A. Benuzzi et al., Phys. Plasmas 5, 2410 (1998).

[28] G. Collins et al., Science 281, 1178 (1998).

[29] A. Benuzzi et al., Phys. Rev. E 54, 2162 (1996).

[30] M. Koenig et al., Appl. Phys. Lett. 72, 1033 (1998).

[31] D. Batani et al., Phys. Rev. B 61, 9287 (2000).

[32] Ya. B. Zel'dovich and Yu. P. Raizer, Physics of Shock Waves and High-Temperature Hydrodynamic Phenomena (Academic Press, New York, 1967).

[33] S. N. Dixit et al., ICF Quarterly Rep. 4, 152 (1994).

[34] P. M. Celliers et al., Appl. Phys. Lett. 73, 1320 (1998).

[35] R. Ramis et al., Comput. Phys. Commun. 49, 475 (1988).

[36] E. Henry et al., Laser Part. Beams 19, 111-115 (2001).

[37] R. F. Trunin, Phys. Usp. 37, 1123 (1994).

[38] A. Morelli, thesis, Università di Milano, 2000 (in Italian, available on request at email address: batani@mib.infn.it). 